\title{
Synthesis and Characterization of DNA Fluorescent Probes Containing a Single Site-Specific Stereoisomer of anti-Benzo[a]pyrene Diol Epoxide- $N^{2}-\mathrm{dG}$
}

\author{
Chao Wang, ${ }^{\dagger}$ Feng Feng, ${ }^{\dagger}$ Zhixin Wang, ${ }^{\dagger}$ Tao $\mathrm{Li}^{\dagger}{ }^{\dagger}$ X. Chris Le, ${ }^{\dagger}$ and Hailin Wang ${ }^{*}{ }^{\dagger}$ \\ State Key Laboratory of Environmental Chemistry and Ecotoxicology, Research Center for Eco-Environmental \\ Sciences, Chinese Academy of Sciences, Beijing 100085, China, and Department of Laboratory Medicine and \\ Pathology, University of Alberta, Edmonton, Alberta T6G 2G3, Canada
}

Received November 8, 2008

\begin{abstract}
We present a comprehensive study of synthesis and characterization of DNA probes containing covalently bound benzo $[a]$ pyrene diol epoxide (BPDE) isomers at a defined site. Short oligonucleotides of 16 mers containing a single trans-(+)- or trans-(-)-anti-BPDE- $N^{2}$-guanine adducts (BPDE-16mer) were first synthesized and then ligated with a fluorescently labeled single-stranded oligonucleotide. The ligation products (double-stranded or single-stranded 90mers) contained a single BPDE adduct of defined stereochemistry and a fluorescent label. The BPDE adduct could be recognized by a specific antibody, and the fluorescent label was useful for highly sensitive laser-induced fluorescence detection. The incorporation of single BPDE in the 16mers was validated by liquid chromatography, UV spectroscopy, and tandem mass spectrometry analysis. The stereochemistry of the single BPDE adducts in the 16mers was further identified by enzyme digestion-coupled stereoselective chromatography analysis. The ligation of BPDE-16mer with normal oligonucleotides for the synthesis of tetramethylrhodamine (TMR)-BPDE90 mers was evaluated. It was found that the modification of the $16 \mathrm{mer}$ by anti-BPDE could significantly reduce the ligation yield of ds90mer and lead to the formation of gapped DNA. The incorporation of a single anti-BPDE adduct in ligated ds90mers was confirmed using an antibody specific to the antiBPDE-dG and affinity capillary electrophoresis. The detection limits of the TMR-BPDE-90mers by capillary electrophoresis coupled with laser-induced fluorescence are below $4 \times 10^{-19} \mathrm{~mol}$.
\end{abstract}

\section{Introduction}

Benzo $[a]$ pyrene, a common environmental pollutant and a representative of carcinogenic polycyclic aromatic hydrocarbons (PAHs), can be metabolized in vivo by cytochrome P450 and epoxide hydrolase to form syn and anti isomers of benzo[a]pyrene-7,8-diol 9,10-epoxide (BPDE) (1). The anti-BPDE is more mutagenic than its syn diastereomer in a series of animal and human cell experiments $(2,3)$. The racemate of anti-BPDE can react with DNA, primarily forming a bulky anti-BPDE- $N^{2}-\mathrm{dG}$ with four stereoisomers of (+)-trans, (-)-trans, (+)-cis, and $(-)$-cis (Scheme 1). The stereochemically different BPDE-DNA adducts may have different repair efficiencies, mutagenicities, and carcinogenicities $(4,5)$. To understand the mechanisms of DNA repair and mutagenesis, ${ }^{32} \mathrm{P}$-labeled probes that contain site-specific BPDE adducts have often been used in previous studies, for example, UvrABC repair (6-9), topoisomerase cleavage $(10,11)$, and translesion synthesis $(12-15)$. Despite the successful applicaitions of ${ }^{32} \mathrm{P}$-labeled probes, fluorescent probes as new alternatives to radioactive probes are desirable. As compared with traditional radioactive labels, fluorescent labels may provide more qualitative and quantitative information, for example, fluorescence intensity, polarization, lifetime, and energy transfer, from which the thermodynamic, kinetic, and conformational properties of protein-DNA interactions can be derived. When the fluorescent probes are combined with

* To whom correspondence should be addressed. Tel/Fax: 0086-1062849600. E-mail: hlwang@rcees.ac.cn.

Chinese Academy of Sciences.

$\div$ University of Alberta.
Scheme 1. Structures of Four anti-BPDE- $N^{2}-\mathrm{dG}$ Stereoisomers

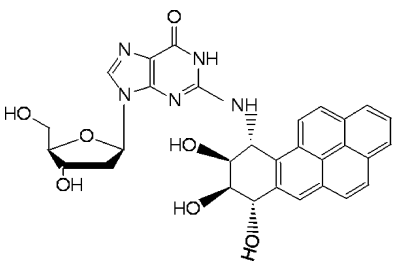

trans-(-)-anti-BPDE- $N^{2}-\mathrm{dG}$

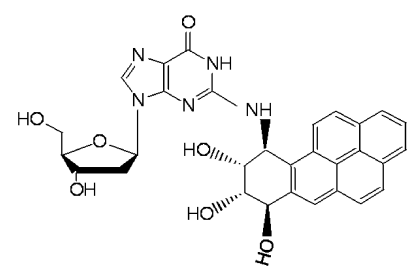

trans-(+)-anti-BPDE- $N^{2}-\mathrm{dG}$

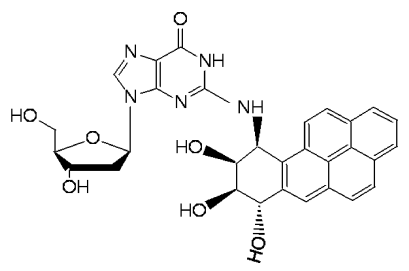

cis-(-)-anti-BPDE- $N^{2}-\mathrm{dG}$

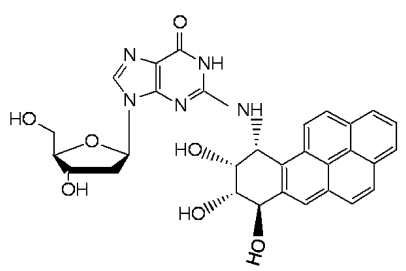

cis-(+)-anti-BPDE- $N^{2}-\mathrm{dG}$ highly sensitive capillary electrophoresis laser-induced fluorescence (CE-LIF), a sensitivity of the zeptomole level and a rapid separation (within several minutes) can be achieved (16).

Because long DNA fragments (approximately a hundred base pairs) may be involved with critical processing of DNA mismatch repair, nucleotide excision repair, and transcription $(17,18)$, the development and application of relatively long damaged DNA fluorescent probes is rational and of emerging interest. Although a few damaged DNA probes have been reported (19), the preparation of relatively long damaged DNA 

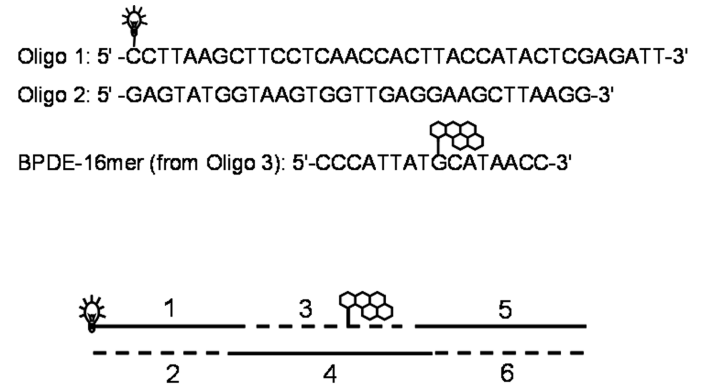

Ligate overnight

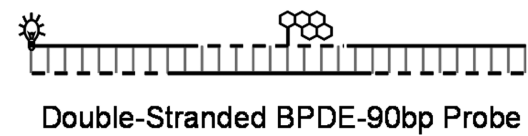

Oligo 4: 5'-CATATGACGGTTATGCATAATGGGAATCTC-3' Oligo 5: 5'-GTCATATGCCGCCTCTGACCTTCCTAGAATTCCATCC-3' Oligo 6: 5'-GGATGGAATTCTAGGAAGGTCAGAGGCGG-3'

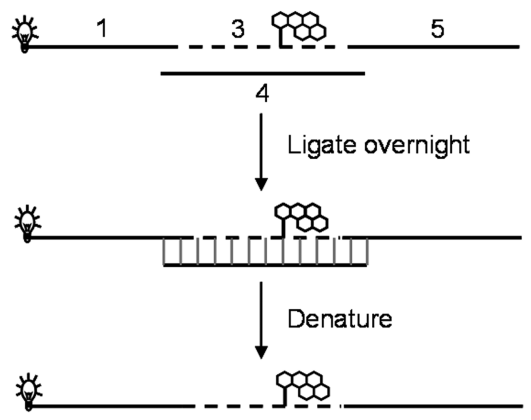

Single-Stranded BPDE-90mer Probe

Figure 1. Schematic illustration of the design and synthesis of TMR-BPDE-ds90mer (left) and TMR-BPDE-ss90mer (right). The 5'-TMR label and a single BPDE adduct are present in the same strand after ligation.

fluorescent probes that have site-specific damage and welldefined stereochemistry is still troublesome and challenging and is short of an updated report for describing the synthesis, characterization, and validation of the BPDE-DNA probes using the latest technologies. In addition, there is no DNA fluorescent probe available containing individual BPDE stereoisomers regardless of the weak native fluorescence of BPDE adduct. In this work, we describe in detail an updated method for the preparation, characterization, and validation of two doublestranded (ds) and two single-stranded (ss) DNA fluorescent probes of 90 mers that each contains a single anti-BPDE- $N^{2}-\mathrm{dG}$ stereoisomer and a fluorescent label.

\section{Experimental Procedures}

Caution: BPDE is carcinogenic and must be handled with care. Chemicals and Reagents. All the tetramethylrhodamine (TMR)labeled and unmodified oligonucleotides were synthesized by TaKaRa Biotech (Dalian, China). Monoclonal mouse anti-BPDE antibody IgG $8 \mathrm{E} 11$ was purchased from Trevigen (Gaithersburg, MD). Bovine serum albumin (BSA) was obtained from Sigma (St. Louis, MO). ( \pm ) $-r-7, t-$ 8-dihydroxy-t-9,10-epoxy-7,8,9,10-tetrahydro-benzo $[a]$ pyrene $[( \pm)$ anti-BPDE] was purchased from the National Cancer Institute Chemical Carcinogen Reference Standard Repository, Midwest Research Institute (Kansas City, MO). T4 DNA ligase, T4 polynucleotide kinase (PNK), calf intestinal alkaline phosphatase, exonuclease I, and ATP were purchased from New England BioLabs (Ipswich, MA). Other chemicals were supplied by Sigma-Aldrich (St. Louis, MO) or Fisher Scientific (Pittsburgh, PA).

Design of Damaged DNA Probes. The BPDE-DNA probes were designed according to our previous work with slight modification (20). The probes contained a fluorescent label at the $5^{\prime}$ termini and a single adduct in the middle of same chain (Figure 1). In the probe synthesis, six or four of the oligonucleotides shown below were involved.

oligo 1: 5'-TMR-CCTTAAGCTTCCTCAACCACTTACCATACTCGAGATT-3'

oligo 2: 5'-GAGTATGGTAAGTGGTTGAGGAAGCTTAAGG-3'

oligo 3: 5'-CCCATTATGCATAACC-3'

$3^{\prime}$

oligo 4: 5'-CATATGACGGTTATGCATAATGGGAATCTC-

oligo 5: 5'-GTCATATGCCGCCTCTGACCTTCCTAGAATTCCATCC-3'

oligo 6: 5'-GGATGGAATTCTAGGAAGGTCAGAGGCGG-3'
These oligonucleotides were initially unphosphorylated. To prepare the stereoisomeric TMR-BPDE-ds90mer, the BPDE-16mer containing a single anti-BPDE- $N^{2}$-dG was first synthesized by direct reaction of unphosphorylated oligo 3 with racemic anti-BPDE and purified as the two isomers of trans-(+)- and trans-(-)-BPDE16 mer. The BPDE-16mer, oligos 2, 4, and 5, were phosphorylated at the $5^{\prime}$ termini and then annealed with unphosphorylated oligo 1 and oligo 6 . The annealed oilgonucleotides were ligated by T4 ligase to form an intact double strand 90mer (Figure 1, left).

The oligonucleotides of oligo 1 , oligo 4 , and phosphorylated BPDE-16mer and oligo 5 were involved with the synthesis of the ss probes (TMR-BPDE-ss90mers, Figure 1, right). In this design, only one short oligo (oligo 4) was used as a complementary strand to assist with the ligation of the oligo 1, BPDE-16mer, and oligo 5 as one strand (Figure 1, right). This design can facilitate the polyacrylamide gel electrophoresis (PAGE) purification of the TMR-BPDE-ss90mers.

Synthesis, Purification, and Characterization of BPDE16mer. The BPDE-16mers were synthesized by the reaction of the unphosphorylated oligo 3 and racemic $( \pm)$-anti-BPDE according to the procedure described previously $(20,21)$. In brief, $400 \mu \mathrm{L}$ of $60 \mu \mathrm{M}$ unphosphorylated oligo 3 solution (dissolved in $20 \mathrm{mM}$ phosphate buffer containing $1.0 \%$ triethylamine, $\mathrm{pH} 11.0$ ) was mixed with $40 \mu \mathrm{L}$ of $3 \mathrm{mM}( \pm)$-anti-BPDE solution (dissolved in freshly prepared tetrahydrofuran/triethylamine, 19:1) and incubated at room temperature over $24 \mathrm{~h}$ in dark.

The synthesized BPDE-16mers were purified on a Hitachi L-2000 series liquid chromatography system from Hitachi (Tokyo, Japan). The analytes were monitored consecutively by a Hitachi L-2455 diode array spectrophotometric detector (DAD) and a Hitachi L-2485 fluorescence detector. The separation was conducted on a column of Capcell Pak Ph UG column (250 mm $\times 4.6 \mathrm{~mm}$ i.d., 5 $\mu \mathrm{m}$ particle size, Shiseido, Tokyo, Japan) with an isocratic elution of the mobile phase that consisted of $7.5 \%$ acetonitrile, $10 \mathrm{mM}$ acetate buffer, and $0.14 \%$ triethylamine $(\mathrm{pH} 7.0)$. The flow rate was $0.75 \mathrm{~mL} / \mathrm{min}$.

The purified BPDE-16mers were identified using HPLC-electrospray ionization (ESI)-quadrupole time-of-flight (Q-TOF)-MS analysis. The instrument consisted of an Alliance 2695 HPLC (Waters, Manchester, United Kingdom) and a Waters Micromass Quattro Micro mass spectrometer equipped with an ESI source. Data acquisition was handled by Mass Lynx 4.0 software (Waters, Milford, MA). The HPLC-MS analysis of BPDE-16mers was conducted using the same column and mobile phase as that for the purification of BPDE-16mers. The flow rate was kept at $0.75 \mathrm{~mL} /$ min for HPLC separation and split into $0.2 \mathrm{~mL} / \mathrm{min}$ for Q-TOF- 
MS analysis. The mass spectrometer was operated in a negative ionization mode. The desolvation temperature and source temperature were set at 200 and $80{ }^{\circ} \mathrm{C}$, respectively. The cone voltage was $20 \mathrm{~V}$, and the capillary voltage was maintained at $2.5 \mathrm{kV}$ for negative mode. Nitrogen was used as a nebulizer gas at a flow rate of $10 \mathrm{~L} / \mathrm{min}$.

Ligation and Purification of TMR-BPDE-90mers. To prepare TMR-BPDE-ss90mer, 170 pmol of oligo 5 and 170 pmol of BPDE16 mer were first phosphorylated at $37^{\circ} \mathrm{C}$ for $2 \mathrm{~h}$ in a $35 \mu \mathrm{L}$ solution containing $1 \times$ ligation buffer and 10 units of T4 PNK. Then, the PNK was denatured by heating at $70{ }^{\circ} \mathrm{C}$ for $10 \mathrm{~min}$. After inactivation of the PNK, the solution was added by two oligonucleotides (170 pmol of oligo 1 and $170 \mathrm{pmol}$ of oligo 4) and 1 $\mathrm{mM}$ ATP and heated at $70{ }^{\circ} \mathrm{C}$ again for $10 \mathrm{~min}$ followed by naturally cooling down at room temperature for annealing oligo 4 with the other oligos. At last, the ligation was initiated by adding 8.5 Weiss units of T4 DNA ligase and carried out overnight at 16 ${ }^{\circ} \mathrm{C}$. The control probe of TMR-ss90mer was synthesized according to the same procedure except that $50 \mathrm{pmol}$ of unmodified $16 \mathrm{mer}$ (oligo 3) was used.

Purification of the TMR-BPDE-ss90mers from the ligation products was carried out on $8 \%$ denaturing PAGE at $200 \mathrm{~V}$ for 80 min. The gel contained $7 \mathrm{M}$ urea. One $\times$ TBE buffer $(90 \mathrm{mM}$ Trisborate, 2 mM EDTA, $\mathrm{pH}$ 8.3) was used for PAGE separation. The bands were visualized by the fluorescence from the TMR label of the oligonucleotides on a visible light transilluminator (Bio-V Company, Xiamen, China) and cut from the gel for recovery. The recovered gel slices were crushed in collect vials and soaked in $300 \mu \mathrm{L}$ of polyacrylamide gel elution buffer $(0.5 \mathrm{~mol} / \mathrm{L}$ Ammonium acetate, $10 \mathrm{mmol} / \mathrm{L}$ Magnesium acetate, $1 \mathrm{mmol} / \mathrm{L}$ EDTA, $\mathrm{pH}$ 8.0), followed by shaking on a rotary shaker in the dark overnight at 37 ${ }^{\circ} \mathrm{C}$. The supernants were aspirated out and transferred into other vials, followed by adding $0.3 \mathrm{vol}$ of $100 \mathrm{mM} \mathrm{MgCl}$ and $2 \mathrm{vol}$ of ice-cold ethanol. The solutions were placed at $-20{ }^{\circ} \mathrm{C}$ for $1.5 \mathrm{~h}$ to facilitate the precipitation of the target products and then centrifuged by $15000 \mathrm{rpm} \times 20 \mathrm{~min}$ at $4{ }^{\circ} \mathrm{C}$. The pellets (target probe, TMRBPDE-ss90mers) were collected and washed once with $70 \%$ ethanol. The collected pellets were dried in the air and redissolved in $\mathrm{ddH}_{2} \mathrm{O}$.

The phosphorylation and ligation in the synthesis of TMR-BPDEds90mers were followed using the same procedure as described for TMR-BPDE-ss90mers except that more oligonucleotides were involved (Figure 1). In this case, BPDE-16mer, oligo 2, oligo 4, and oligo 5 (with the molar ratio of $1: 1$ ) were required to be phosphorylated prior to the ligation. The ligation products were purified using $12 \%$ native PAGE. The other procedures were the same as that for the purification of TMR-BPDE-ss90mers.

Identification of TMR-BPDE-90mers by Affinity Capillary Electrophoresis (ACE). The purifed TMR-BPDE-90mers were further identified by ACE using anti-BPDE mouse monoclonal antibody 8E11 as an affinity probe. The ACE analysis was carried out using a laboratory-built CE-LIF system as described previously (22). A green $\mathrm{He}-\mathrm{Ne}$ laser beam $(543.5 \mathrm{~nm}, 1 \mathrm{~mW}$, Melles Griot, Irvine, CA) was used for exciting TMR label, and the fluorescence was filtered through a band-pass of $575 \mathrm{~nm}$ (Semrock, Rochester, NY) and detected by a photomultiplier tube (PMT, model R3896, Hamamatsu Photonics, Japan). The data were acquired with a frequency of $20 \mathrm{~Hz}$ and handled by a HW-2000 chromatographic workstation (Qianpu Software Co., Shanghai, China). Uncoated fused silica capillary (Yongnian Optical Fiber Co., Hebei, China) with a dimension of $25 \mu \mathrm{m}$ i.d. $\times 365 \mu \mathrm{m}$ o.d. and $35 \mathrm{~cm}$ long (effective length, $30 \mathrm{~cm}$ ) was used throughout the experiments. Samples were electrokinetically injected into the capillary by applying an injection voltage of $15 \mathrm{kV}$ for $5 \mathrm{~s}$. Each sample was repeatedly injected for three times. The separation was carried out by applying a voltage of $15 \mathrm{kV}$ at room temperature. The sample and running buffers were $2 \times$ TGA $(\mathrm{pH} 7.5,14 \mathrm{mM}$ Tris, $108 \mathrm{mM}$ glycine, and HAc $\sim 10.5 \mathrm{mM})$ and $1 \times \mathrm{TG}(\mathrm{pH} 8.5,30 \mathrm{mM}$ Tris and $160 \mathrm{mM}$ glycine), respectively.

Prior to ACE analysis, the stock solutions of TMR-BPDE-90mers were diluted to an appropriate concentration in the buffer of $2 \times$

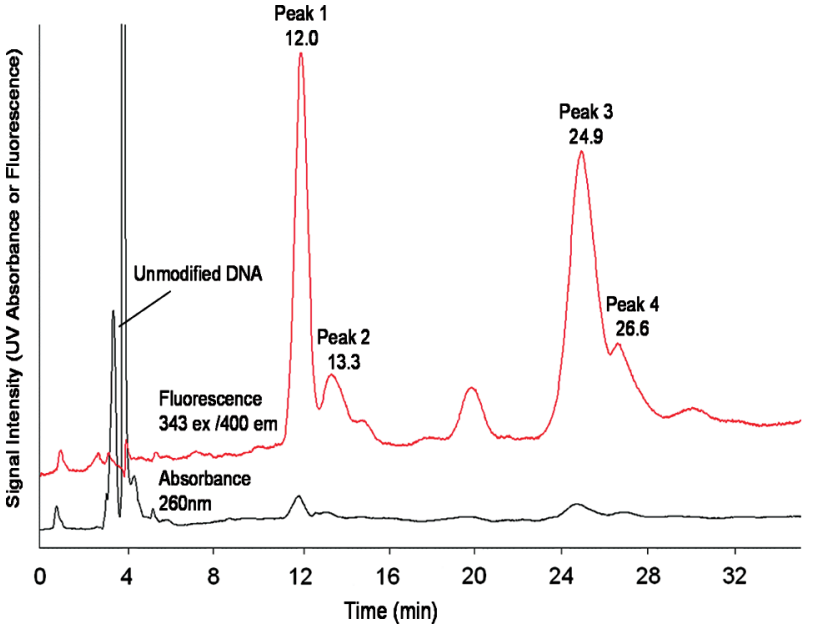

Figure 2. Chromatograms obtained from HPLC analysis of the reaction products of $( \pm)$-anti-BPDE and $16 \mathrm{mer}$. The eluted species included unmodified 16mer ( $3.9 \mathrm{~min})$ and four stereoisomers of anti-BPDE16 mer (peak 1, 12.0 min; peak 2, 13.3 min; peak 3, 24.9 min; and peak 4, $26.6 \mathrm{~min})$.

TGA. The DNA probes were denatured by heating at $95{ }^{\circ} \mathrm{C}$ for 5 min in a heating block and then transferred directly to ice for 10 min to prevent their reannealing. The diluted probe solutions were further mixed with proper concentrations of TMR and mAb 8E11 and $100 \mu \mathrm{g} / \mathrm{mL}$ BSA. The sample was gently vortexed for $5 \mathrm{~s}$ and incubated at room temperature for $30 \mathrm{~min}$ before CE-LIF analysis. In the experiments, TMR was used as an internal standard to correct variations in the injection volume, and BSA was used to enhance the formation and stability of the immuno-complexes (23).

\section{Results and Discussion}

Purification and Characterization of BPDE-16mer Stereoisomers. The stereoisomers of BPDE-16mers were generated from the overnight reaction of $( \pm$-anti-BPDE and the $16 \mathrm{mer}$ (oligo 3). Besides the generated adducts (BPDE-16mers), the reaction mixture also contained excess unreacted $16 \mathrm{mer}$ and BPDE tetrols, which were the hydrolysis byproducts of racemic anti-BPDE. Figure 2 shows the chromatograms from HPLC analysis of the reaction products. The unreacted 16mer migrated out first (3.9 min), followed by the four BPDE-16mer DNA adducts (12.0, 13.3, 24.9, and $26.6 \mathrm{~min})$. The hydrolysis byproducts (BPDE tetrols) were not eluted out under the chosen HPLC conditions. The unreacted $16 \mathrm{mer}$ only has strong UV absorbance at $260 \mathrm{~nm}$ but does not have detectable fluorescence response (Figure 2). The four stereoisomers of BPDE-16mer have the spectroscopic characteristics of both BPDE (248-250, 332-334, and 348-350 nm) and DNA (259-260 nm) (Supporting Information, Figure S1) and yield strong fluorescence response by excitation of $343 \mathrm{~nm}$ and emission of $400 \mathrm{~nm}$ (Figure 2). To test the purity of the separated BPDE-16mer, the fractions of the two major stereoisomers of the BPDE-16mer (12.0 and $24.9 \mathrm{~min}$, Figure 2) were collected separately and were further analyzed using HPLC. The obtained chromatograms showed the presence of only one peak corresponding to the single stereoisomer of BPDE-16mer for each purified fraction (data not shown). The two major stereoisomers of the BPDE-16mer were identified as trans-(-)-anti-BPDE- $N^{2}-\mathrm{dG}$ $16 \mathrm{mer}$ (eluting at $12.0 \mathrm{~min})$ and trans- $(+)$-anti-BPDE- $N^{2}-\mathrm{dG}-$ $16 \mathrm{mer}$ (eluting at $24.9 \mathrm{~min}$ ) as described below.

The incorporation of single anti-BPDE adduct in the BPDE16 mers was confirmed by HPLC-ESI-Q-TOF-MS analysis (see Figure $3 \mathrm{~A}-\mathrm{C}$ ). The unreacted $16 \mathrm{mer}$ displays a $\mathrm{m} / \mathrm{z}$ of 4783 amu (Figure 3A), and both stereoisomers of the BPDE-16mer 


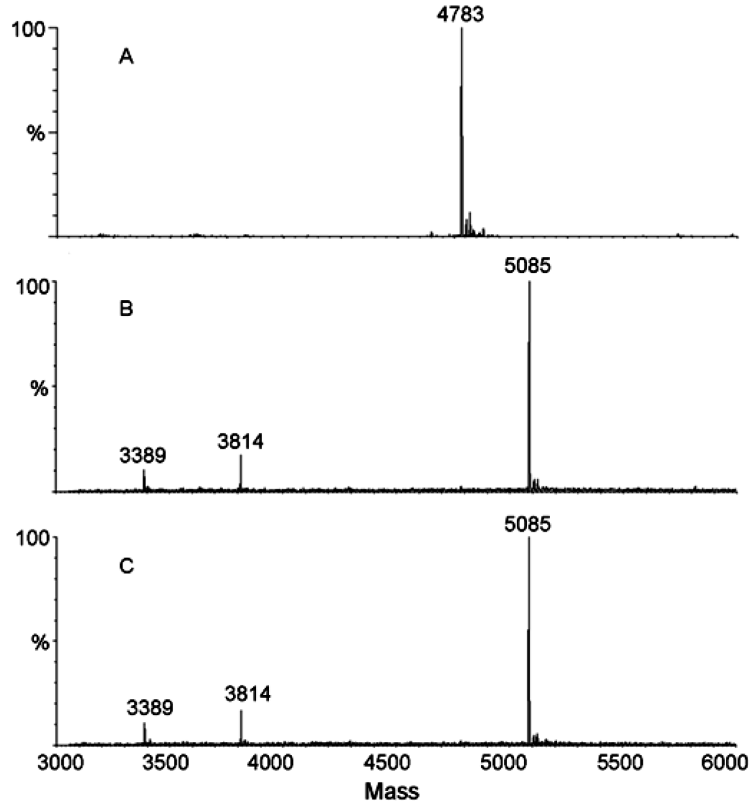

Figure 3. Characterization of the BPDE-16mer by HPLC-Q-TOF-MS. Ion spectra $\mathrm{A}-\mathrm{C}$ were obtained from unmodified $16 \mathrm{mer}$ and two major BPDE-16mer isomers (peaks 1 and 3 in Figure 2). The chromatogram is not shown.

show a major peak at $\mathrm{m} / \mathrm{z}$ of 5085 amu (Figure 3B,C). The increased mass shift of $302 \mathrm{Da}$ is consistent with the presence of a single BPDE in the 16 mer. Two additional fragment peaks of $\mathrm{m} / \mathrm{z}, 3389$ and $\mathrm{m} / \mathrm{z} 3814$ were observed for the two stereoisomers of the BPDE-16mer (Figure 3B,C). This was not observed for the unmodified 16 mer in the same mass range of 3000-6000 Da. These results suggest that the incorporation of a single BPDE adduct into the 16mer may decrease the stability of the precursor molecular ions (BPDE-16mer) during the ESI process.

The stereochemistry and the binding site of the BPDE adduct in the two purified BPDE-16mer stereoisomers were further identified by enzyme digestion-coupled stereoselective chromatography analysis (refer to the Supporting Information, Figure S2). In the case of HPLC stereoselective analysis, four standard stereoisomers of anti-BPDE- $N^{2}-\mathrm{dG}$ were used. The stereochemistry of the four standard anti-BPDE- $N^{2}-\mathrm{dG}$ stereoisomers has been previously identified and confirmed by HPLC-DAD-FL, HPLC/ESI-Q-TOF-MS/MS, and circular dichroism (CD) (24). The enzymatic digest of the first BPDE-16mer fraction (peak 1 , Figure 2) is overlapped with trans-(-)-anti-BPDE- $N^{2}-\mathrm{dG}$ (Supporting Information, Figure S2, trace 2). Likewise, the enzymatic digest of the third BPDE-16mer fraction (peak 3, Figure 2) is overlapped with trans-(+)-anti-BPDE- $N^{2}$-dG (Supporting Information, Figure S2, traces 3 ). Therefore, the two major anti-BPDE-16mers collected from peak 1 and peak 3 in Figure 2 are trans-(-)-anti-BPDE- $N^{2}-\mathrm{dG}-16$ mer [trans-(-)BPDE-16mer] and trans-(+)-anti-BPDE- $N^{2}$-dG-16mer [trans$(+)$-BPDE-16mer], respectively. The observed predominant formation of the trans-anti-BPDE- $N^{2}-\mathrm{dG}$ adducts in the reaction of the $16 \mathrm{mer}$ and racemic anti-BPDE is consistent with the previous studies $(21,25)$.

Synthesis and Purification of ss and ds TMR-BPDE90mers. Two types of DNA damaged probes, ss and ds TMRBPDE-90mers (TMR-BPDE-ss90mer and TMR-BPDE-ds90mer), were synthesized through the ligation as described in the Experimental Procedures. Typical separation of the TMRBPDE-ss90mer ligation products on a denaturing polyacrylamide gel (7 M urea, 8\% PAGE) is shown in Figure 4. Two major

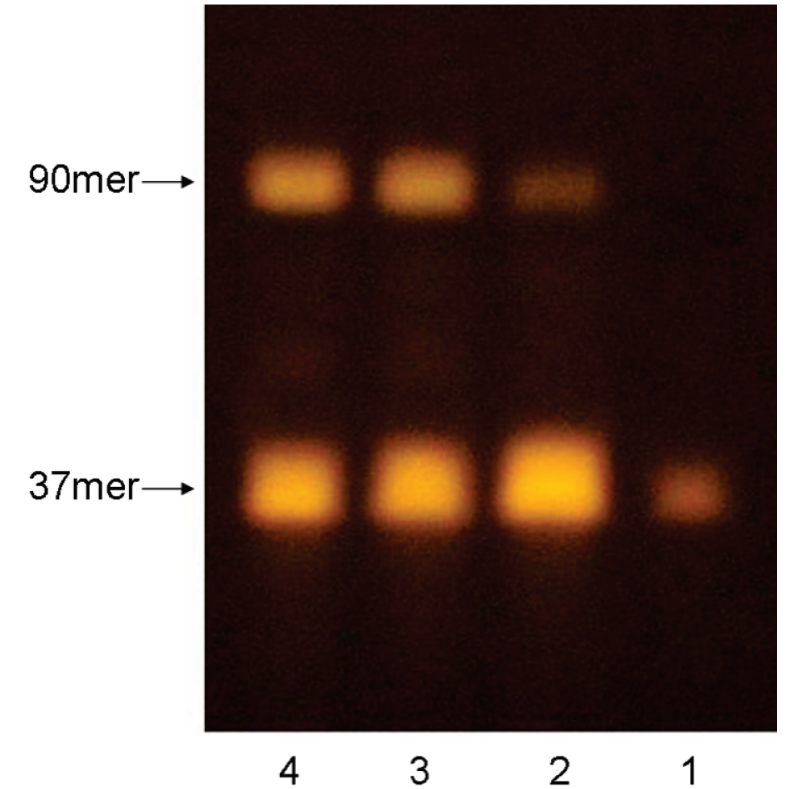

Figure 4. Gel electrophoresis separation of the ligation products of ss 90mers using $8 \%$ denaturing PAGE (7 M urea) included. Lane 1, TMR37 mer (oligo 1); lane 2, ligation products of control TMR-ss90mer using undamaged $16 \mathrm{mer}$; lane 3 , ligation products of TMR-BPDE-ss90mer using trans-(-)-BPDE-16mer; and lane 4, ligation products of TMRBPDE-ss90mer using trans-(+)-BPDE-16mer.

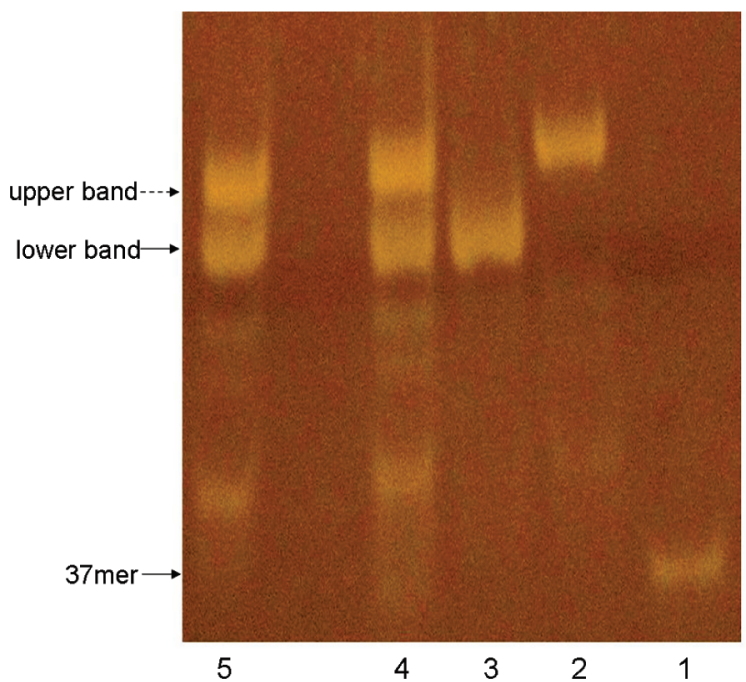

Figure 5. Gel electrophoresis separation of the ligation products of ds 90 mers using $12 \%$ native PAGE. In all ligations, the amount of each oligonucleotide was 200 pmol. Lane 1, only TMR-37mer; lane 2, ligation products of TMR-gapped-ds90mer (missing a 16mer); lane 3, ligation products of control TMR-ds90mer using undamaged 16mer; lane 4, ligation products of TMR-BPDE-ds90mer using trans-(-)BPDE-16mer; and lane 5, ligation products of TMR-BPDE-ds90mer using trans-(+)-BPDE-16mer.

bands correspond to TMR-BPDE-ss90mer product and unligated 37mer (lanes 3 and 4, Figure 4). No migration difference was found between the two TMR-BPDE-ss90mers (lanes 3 and 4), and the control TMR-ss90mer (lane 2) was observed under our denaturing PAGE conditions, although conformation of undamaged 90 mer and the damaged 90mer may be slightly different. In addition, several minor bands were observed in lanes 2-4, indicating that partial ligation products were formed (Figure 4).

The ligation products of TMR-BPDE-ds90mers were separated on a $12 \%$ native PAGE. The PAGE images from the TMRBPDE-ds90mer preparation are illustrated in Figure 5. The complete ligation of six undamaged oligonucleotides (in same molar concentration) results in the formation of undamaged 
TMR-ds90mer, which as expected has a slower migration (lane 3 , Figure 5) than that of the TMR-37mer (lane 1, Figure 5). The ligation products from the trans-(+)- and trans-(-)-BPDE16mer with the other five oligos (lanes 4 and 5, Figure 5) appear as two major bands. The same mixed oligos cannot generate the two major bands if no ligation is involved (data not shown), suggesting that the two major bands are ligation products. The lower major bands (indicated with a solid arrow) have the same migration position as that of the TMR-ds90mer, suggesting that it is TMR-BPDE-ds90mer. The identity of TMR-BPDEds90mers is further confirmed by affinity CE using specific monoclonal antibody as an affinity probe (see the later description). The identity of the upper bands that have slower migration (indicated with a dashed arrow) is not clear. We speculated that the upper band might be a gapped TMR-ds90mer, which missed the central 16mer section. To support this speculation, we conducted a modified ligation by omitting the BPDE-16mer in the ligation reaction. The PAGE analysis shows a major band (lane 2, Figure 5) with the same migration position as that of the upper band in lanes 4 and 5 (Figure 5). These results suggest that the upper band in lanes 4 and 5 is the gapped TMRds90mer. The slower migration of the gapped TMR-ds90mer than normal TMR-ds90mer suggests that the missing of $16 \mathrm{mer}$ causes an abnormal conformation of the dsDNA and retards its electrophoretic mobility in the gel. The ACE analysis further confirms that the prepared probes from the upper bands do not contain an anti-BPDE moiety (described later).

The ligation yields of the control TMR-ds90mer and TMRBPDE-ds90mers are very different. In all of the ligations for the generation of ds90mers, the oligonucleotides were used in equal molar concentrations. We observed that the ligation of normal six oligonucleotides would generate more than $80 \%$ ds90mer (lane 2, Figure 5), but the ligation of either stereoisomer of the BPDE-16mer with other normal five oligonucleotides just generated less than $50 \%$ target ds 90 mer and a byproduct of a gapped ds90mer ( 40\%) (lanes 4 and 5, Figure 5). Previous studies have shown that the covalently bound BPDE in oligonucleotides decreased the thermal stability of ds DNA (25). In the case of trans- $( \pm)$-BPDE-11mer duplexes, the characteristic duplexes melting points $\left(T_{\mathrm{m}}\right)$ were lower by $8-10^{\circ} \mathrm{C}$ than that of the undamaged duplexes $\left(51^{\circ} \mathrm{C}\right)$. In our experiments, the existence of BPDE in 16mer probably hindered its correct annealing with the complementary oligo, leading to the decreased yield of the target TMR-BPDE-90mers and the formation of the gapped TMR-ds90mer. It has been shown that ligation efficiency is approximately $17 \%$ for a short damaged 4 mer even by the use of $200 \times$ excess damaged oligo (26) and $17-40 \%$ for a damaged 16 mer with a BPDE adduct near the $5^{\prime}$ termini $(27,28)$, respectively. In our experiment, the use of a 16 mer with BPDE-dG in the middle of chain can recover the reduced ligation efficiency to certain degree $(\sim 50 \%)$.

ACE Reveals the Incorporation of anti-BPDE- $\mathrm{N}^{2}-\mathrm{dG}$ in the Damaged DNA Probes. Our previous work demonstrated that the mouse monoclonal antibody mAb 8E11 could bind specifically to the BPDE-DNA adducts but not undamaged DNA $(20,29)$. By taking advantage of the highly specific recognition of anti-BPDE-dG by mAb 8E11, we further determined whether the anti-BPDE- $N^{2}-\mathrm{dG}$ was incorporated into the TMR-BPDE90mers.

Figure 6 shows electropherograms from the ACE analysis of two stereoisomeric TMR-BPDE-ss90mers. In these experiments, excess mAb 8E11 over the probes (33-66 vs $5 \mathrm{nM}$ ) was used. A complex peak (peak 2) was observed when the damaged probes were incubated with mAb 8E11 (lower traces
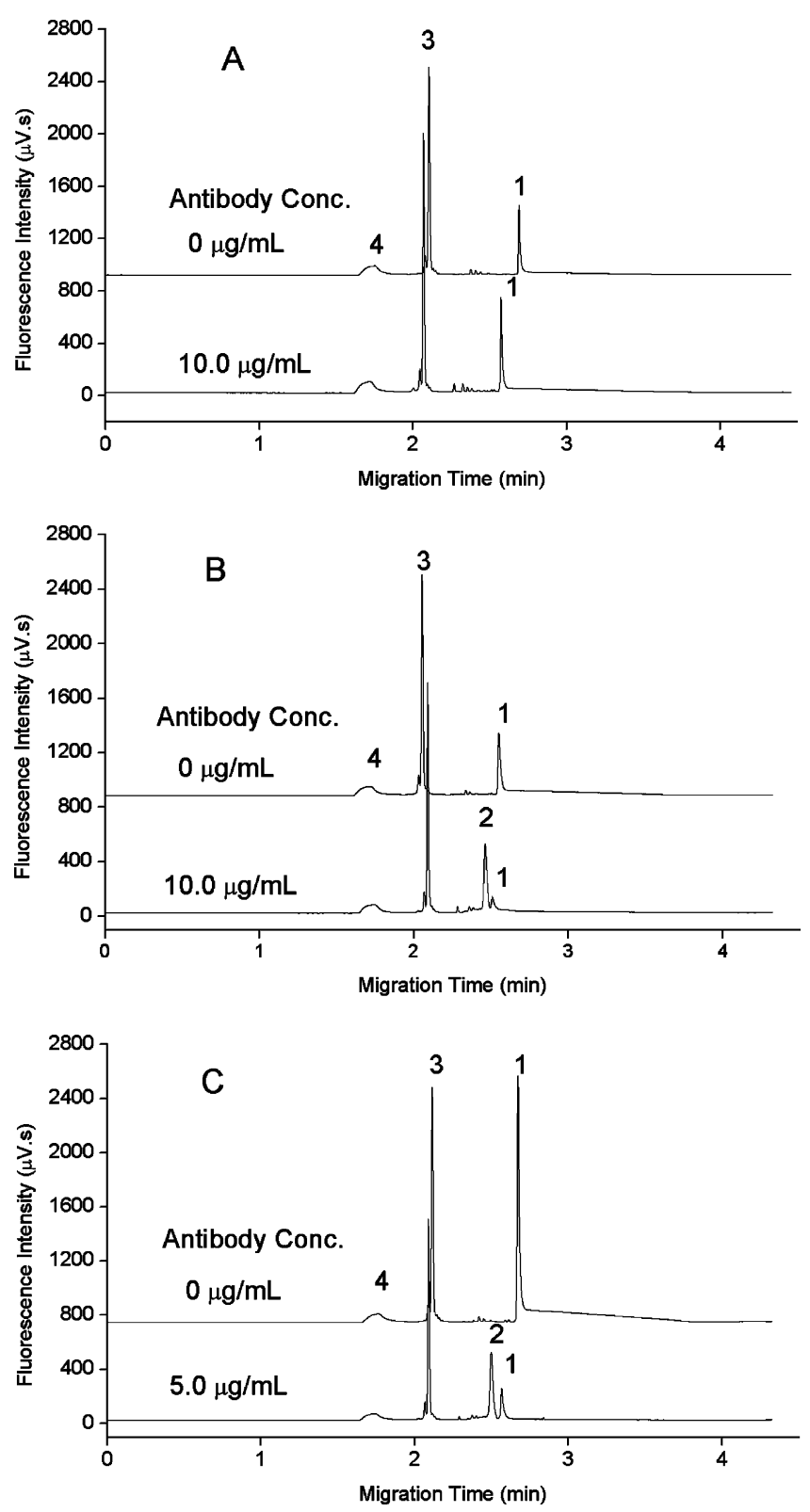

Figure 6. ACE identification of the TMR-BPDE-ss90mer probes using specific mAb 8E11. (A) Control TMR-ss90mer, (B) TMR-trans-(-)BPDE-90mer, and (C) TMR-trans-(+)-BPDE-90mer. The probe $(5 \mathrm{nM})$ was incubated with mAb 8E11 (5 or $10 \mu \mathrm{g} / \mathrm{mL})$ for $30 \mathrm{~min}$. Peak 1,2 , 3 , and 4 correspond to the unbound probe, the complex of the probe and $\mathrm{mAb} 8 \mathrm{E} 11$, TMR, and an impurity of TMR, respectively.

in Figure 6B,C). The complex peak of mAb 8E11 and TMRBPDE-ss90mer (peak 2) was well-resolved from the unbound TMR-BPDE-ss90mer (peak 1). In all electropherograms, the complexes of mAb 8E11 and the probes have faster migration than the unbound probes. The faster migration of the complexes is consistent with their decreased charge/mass ratio when compared with the unbound TMR-BPDE-ss90mers. Approximately $66.2 \%$ of trans-(+)- and $75.7 \%$ of trans-(-)-TMRBPDE-ss90mers are bound to $\mathrm{mAb} 8 \mathrm{E} 11$. There is no complex between the mAb 8E11 and the undamaged TMR-ss90mer (Figure 6A), confirming that $\mathrm{mAb} 8 \mathrm{E} 11$ is specifically bound to the BPDE moiety of the two stereoisomeric TMR-BPDEss90mers. These results also validate the incorporation of antiBPDE- $N^{2}-\mathrm{dG}$ in both stereoisomeric TMR-BPDE-90mers but not in the undamaged 90mer (Figure 6).

We further examined the incorporation of anti-BPDE- $N^{2}-\mathrm{dG}$ in the ligation products for synthesis of TMR-BPDE-ds90mers. 

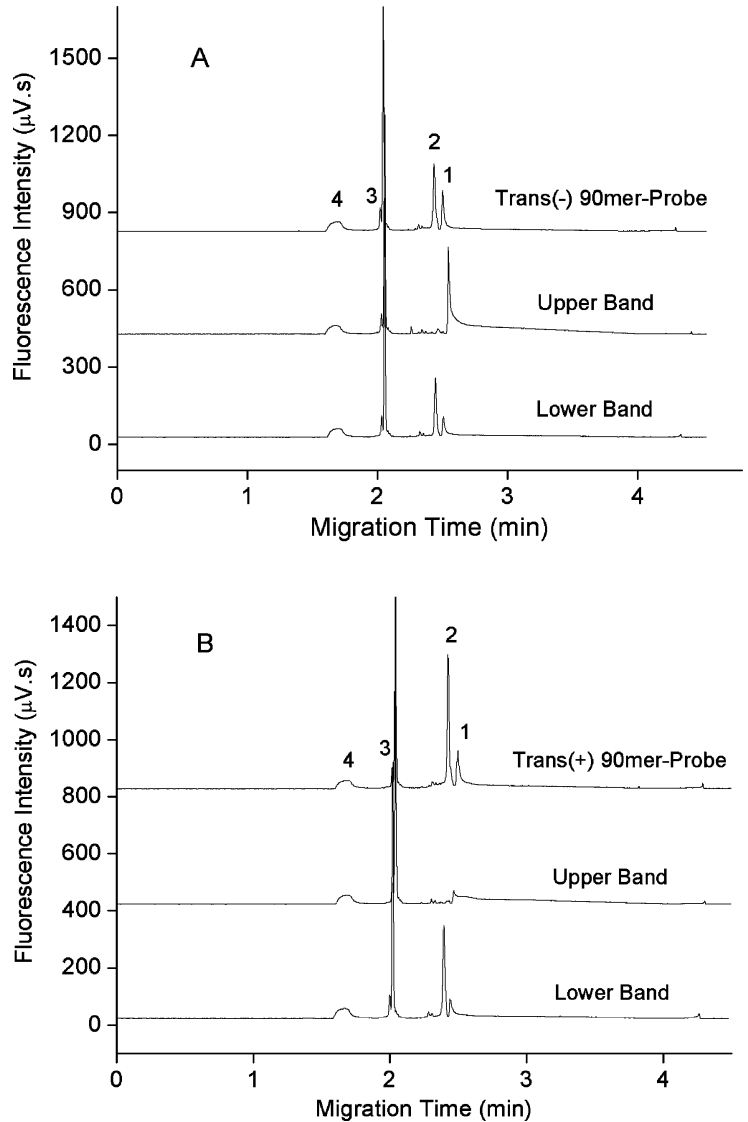

Figure 7. ACE identification of the TMR-BPDE-ds90mer probes using specific mAb 8E11. (A) trans-(-)-BPDE-ds90mer ligation products and (B) trans-(+)-BPDE-ds90mer ligation products. The ds90mers were denatured into ss 90mers $(5 \mathrm{nM})$ and incubated with mAb 8E11 (3.3 $\mathrm{nM}$ ) for $30 \mathrm{~min}$. Peaks $1,2,3$, and 4 correspond to unbound $90 \mathrm{mer}$ probe, complex of 90 mer probe with mAb 8E11, TMR, and an impurity of TMR, respectively.

The ligation products recovered from the upper and lower bands (indicated with arrows, Figure 5) were denatured and then incubated with $\mathrm{mAb} 8 \mathrm{E} 11$. The ACE analysis (Figure 7) shows that the purified products collected from the lower bands could form the complex with mAb 8E11. Approximately $80.8 \%$ of the ligation product that forms the lower band of lane 4 in Figure 5 was bound to mAb $8 \mathrm{E} 11$ (Figure 7A, bottom trace). Likewise, $70.9 \%$ of the ligation product recovered from the lower band of lane 5 in Figure 5 was bound to mAb 8E11 (Figure 7B, bottom trace). There was no complex (Figure 7A,B, middle trace) between mAb 8E11 and either product collected from the upper band in lane 4 or 5 of Figure 5. Nondenatured TMRBPDE-ds90mers could not form an observable complex with mAb 8E11 (data not shown). These results confirm that our procedures were successful in the preparation and identification of the TMR-BPDE-ds90mer.

The stereoisomeric TMR-BPDE-90mer probes prepared in this work have potential applications in the study of site-specific lesion mutagenesis, polymerase bypass, and DNA repair and their stereoselectivity, as well as in developing ultrasensitive assay for detection of trace BPDE-DNA adducts (30). By taking advantage of fluorescence properties (e.g., fluorescence polarization and fluorescence resonance energy transfer), the fluorescent tag in the prepared damaged DNA fluorescent probes may provide dynamic information on the protein-DNA interactions while traditional radioactive DNA damage probes only provide the information on radioactive intensity. In addition, CE-LIF analysis of dye-labeled DNA probes based on a laboratory-built instrument can provide high detection sensitivity at subattomole levels $(23,31,32)$. In this work, the detection limits of TMRBPDE-90mers by CE-LIF are as low as $50 \mathrm{pM}$ in concentration and $4 \times 10^{-19} \mathrm{~mol}$ in mass.

\section{Conclusion}

In this work, we report the comprehensive study on the synthesis and characterization of both ss and ds BPDE-DNA fluorescent probes containing specific stereoisomers of BPDE. The BPDE-DNA probes have a single adduct with well-defined stereochemistry and carry a fluorescent label for highly sensitive LIF detection. These procedures could be used for the synthesis of longer DNA probes, by using longer flanking oligonucleotides. The purified and well-characterized DNA probes will be further used to investigate the affinity of the antibody and its stereochemical recognition of anti-BPDE- $N^{2}-\mathrm{dG}$ DNA adducts and will also be used to optimize the CE-LIF assays for the detection of the BPDE-DNA adducts. In addition, the synthesized long damaged DNA probes can be used to study DNA-protein interactions and their roles in DNA replication, transcription, recombination, repair, and translesion synthesis.

Acknowledgment. This work was supported by Grants from the National Natural Science Foundation of China (Nos. 20737003, 20677066, and 20621703), the National Basic Research Program of China (Nos. 2007CB407305 and 2008CB417201), and the Chinese Academy of Sciences (YZ200749) to H.W.

Supporting Information Available: Figures of UV absorption spectra of the four stereoisomeric BPDE-16mers and identification of the adduct stereochemistry of BPDE-16mers using enzyme digestion and HPLC-DAD-FL analysis. This material is available free of charge via the Internet at http:// pubs.acs.org.

\section{References}

(1) Xue, W., and Warshawsky, D. (2005) Metabolic activation of polycyclic and heterocyclic aromatic hydrocarbons and DNA damage: A review. Toxicol. Appl. Pharmacol. 206, 73-93.

(2) Newbold, R. F., and Brookes, P. (1976) Exceptional mutagenicity of a benzo[a]pyrene diol epoxide in cultured mammalian cells. Nature $261,52-54$.

(3) Huberman, E., Sachs, L., Yang, S. K., and Gelboin, H. V. (1976) Identification of mutagenic metabolites of benzo[a]pyrene in mammalian cells. Proc. Natl. Acad. Sci. U.S.A. 73, 607-611.

(4) Friedberg, E. C., Walker, G. C., and Siede, W. (1995) DNA Repair and Mutagenesis, ASM Press, Washington, DC.

(5) Geacintov, N. E., Cosman, M., Hingerty, B. E., Amin, S., Broyde, S., and Patel, D. J. (1997) NMR solution structures of stereoisomeric covalent polycyclic aromatic carcinogen-DNA adducts: Principles, patterns, and diversity. Chem. Res. Toxicol. 10, 111-146.

(6) Hoare, S., Zou, Y., Purohit, V., Krishnasamy, R., Skorvaga, M., Van Houten, B., Geacintov, N. E., and Basu, A. K. (2000) Differential incision of bulky carcinogen-DNA adducts by the UvrABC nuclease: Comparison of incision rates and the interactions of Uvr subunits with lesions of different structures. Biochemistry 39, 12252-12261.

(7) Zou, Y., Shell, S. M., Utzat, C. D., Luo, C., Yang, Z., Geacintov, N. E., and Basu, A. K. (2003) Effects of DNA adduct structure and sequence context on strand opening of repair intermediates and incision by UvrABC nuclease. Biochemistry 42, 12654-12661.

(8) Jiang, G. H., Skorvaga, M., Croteau, D. L., Van Houten, B., and States, J. C. (2006) Robust incision of Benoz[a]pyrene-7,8-dihyrodiol-9,10epoxide-DNA adducts by a recombinant thermoresistant interspecies combination UvrABC endonuclease system. Biochemistry 45, 78347843.

(9) Ruan, Q., Liu, T., Kolbanovskiy, A., Liu, Y., Ren, J., Skorvaga, M., Zou, Y., Lader, J., Malkani, B., Amin, S., Van Houten, B., and Geacintov, N. E. (2007) Sequence context- and temperaturedependent nucleotide excision repair of a benzo[a]pyrene diol 
epoxide-guanine DNA adduct catalyzed by thermophilic UvrABC proteins. Biochemistry 46, 7006-7015.

(10) Pommier, Y., Kohlhagen, G., Pourquier, P., Sayer, J. M., Kroth, H., and Jerina, D. M. (2000) Benzo[a]pyrene diol epoxide adducts in DNA are potent suppressors of a normal topoisomerase I cleavage site and powerful inducers of other topoisomerase I cleavages. Proc. Natl. Acad. Sci. U.S.A. 97, 2040-2045.

(11) Khan, Q. A., Kohlhagen, G., Marshall, R., Austin, C. A., Kalena, G. P., Kroth, H., Sayer, J. M., Jerina, D. M., and Pommier, Y. (2003) Position-specific trapping of topoisomerase II by benzo[a]pyrene diol epoxide adducts: Implications for interactions with intercalating anticancer agents. Proc. Natl. Acad. Sci. U.S.A. 100, 12498-12503.

(12) Suzuki, N., Ohashi, E., Kolbanovskiy, A., Geacintov, N. E., Grollman, A. P., Ohmori, H., and Shibutani, S. (2002) Translesion synthesis by human DNA polymerase $\kappa$ on a DNA template containing a single stereoisomer of dG-(+)- or dG-(-)-anti- $N^{2}$-BPDE (7,8-dihydroxy-anti9,10epoxy-7,8,9,10-tetrahydro benzo[a]pyrene). Biochemistry 41, 6100 6106.

(13) Chiapperino, D., Kroth, H., Kramarczuk, I. H., Sayer, J. M., Masutani, C., Hanaoka, F., Jerina, D. M., and Cheh, A. M. (2002) Preferential misincorporation of purine nucleotides by human DNA polymerase $\eta$ opposite benzo[a]pyrene 7,8-diol 9,10-epoxide deoxyguanosine adducts. J. Biol. Chem. 277, 11765-11771.

(14) Rechkoblit, O., Zhang, Y., Guo, D., Wang, Z., Amin, S., Krzeminsky, J., Louneva, N., and Geacintov, N. E. (2002) Translesion synthesis past bulky benzo[a]pyrene diol epoxide $N^{2}-\mathrm{dG}$ and $N^{6}-\mathrm{dA}$ lesions catalyzed by DNA bypass polymerases. J. Biol. Chem. 277, 3048830494.

(15) Huang, X., Kolbanovskiy, A., Wu, X., Zhang, Y., Wang, Z., Zhuang, P., Amin, S., and Geacintov, N. E. (2003) Effects of base sequence context on translesion synthesis past a bulky (+)-trans-anti-B[a]P$N 2$-dG lesion catalyzed by the y-family polymerase pol $\kappa$. Biochemistry 42, 2456-2466.

(16) Wang, H., Lu, M., and Le, X. C. (2005) DNA-driven focusing for protein-DNA binding assays using capillary electrophoresis. Anal. Chem. 77, 4985-4990.

(17) Mocquet, V., Kropachev, K., Kolbanovskiy, A., Tapias, A., Cai, Y., Broyde, S., Geacintov, N. E., and Egly, J.-M. (2007) The human DNA repair factor XPC-HR23B distinguishes stereoisomeric benzo[a]pyrenyl-DNA lesions. EMBO J. 26, 2923-2932.

(18) Yang, W. (2008) Structure and mechanism for DNA lesion recognition. Cell Res. 18, 184-197.

(19) James, C. D., and Essigmann, J. M. (2008) Biological properties of single chemical-DNA adducts: A twenty year perspective. Chem. Res. Toxicol. 21, 232-252.

(20) Carnelley, T. J., Barker, S., Wang, H., Tan, W. G., Weinfeld, M., and Le, X. C. (2001) Synthesis, characterization, and applications of a fluorescent probe of DNA damage. Chem. Res. Toxicol. 14, 15131522.

(21) Feng, F., and Wang, H. (2007) Simultaneous analysis of four stereoisomers of anti-benzo[a]pyrene diol epoxide-deoxyguanosine adducts in short oligodeoxynucleotides using reversed-phase highperformance liquid chromatography. J. Chromatogr. A 1162, 141148.

(22) Wang, Z., Wang, C., Yin, J., Li, T., Song, M., Lu, M., and Wang, H. (2008) Focusing and stabilization of bis-intercalating dye-DNA complexes for high sensitive capillary electrophoresis-laser induced fluorescence DNA analysis. Electrophoresis 29, 4454-4462.

(23) Wang, H., Lu, M., Weinfeld, M., and Le, X. C. (2003) Enhancement of immunocomplex detection and application to assays for DNA adduct of benzo[a]pyrene. Anal. Chem. 75, 247-254.

(24) Feng, F., Yin, J., Song, M., and Wang, H. (2008) Preparation, identification and analysis of stereoisomeric anti-benzo[a]pyrene diol epoxide-deoxyguanosine adducts using phenyl liquid chromatography with diode array, fluorescence and tandem mass spectrometry detection. J. Chromatogr. A 1183, 119-128.

(25) Cheng, S. C., Hilton, B. D., Roman, J. M., and Dipple, A. (1989) DNA adducts from carcinogenic and noncarcinogenic enantiomers of benzo[a]pyrene dihydrodiol epoxide. Chem. Res. Toxicol. 2, 334-340.

(26) Benasutti, M., Z., Ezzedine, D., and Loechler, E. L.,. (1988) Construction of an Escherichia coli vector containing the major DNA adduct of activated benzo[a]pyrene at a defined site. Chem. Res. Toxicol. 1, 160-168.

(27) Page, J. E., Pilcher, A. S., Yagi, H., Sayer, J. M., Jerina, D. M., and Anthony, D. (1999) Mutational consequences of replication of M13mp7L2 constructs containing cis-opened benzo[ $a$ ]pyrene 7,8diol 9,10-epoxide-deoxyadenosine adducts. Chem. Res. Toxicol. 12, 258-263.

(28) Pontn, I., Kroth, H., Sayer, J. M., Dipple, A., and Jerina, D. M. (2001) Differences between the mutational consequences of replication of cis- and trans-opened benzo[a]pyrene 7,8-diol 9,10-epoxide-deoxyguanosine adducts in M13mp7L2 constructs. Chem. Res. Toxicol. 14, 720-72.

(29) Wang, H., Lu, M., Mei, N., Lee, J., Weinfeld, M., and Le, X. C. (2003) Immunoassays using capillary electrophoresis laser induced fluorescence detection for DNA adducts. Anal. Chim. Acta 500, 13-20.

(30) Wang, C., Wang, Z., Feng, F., Yin, J., Song, M., and Wang, H. Stereospecific binding of monoclonal antibody to anti-BPDE- $N^{2}$ $\mathrm{dG}$ adducts in single nucleosides and oligonucleotides studied by affinity capillary electrophoresis. Manuscript in preparation.

(31) Song, M. Y., Zhang, Y. X., Li, T., Wang, Z. X., Yin, J. F., and Wang, H. (2009) Highly sensitive detection of human thrombin in serum by affinity capillary electrophoresis/laser-induced fluorescence polarization using aptamers as probes. J. Chromatogr. A 1216, 873-838.

(32) Chen, D. Y., and Dovichi, N. J. (1996) Single-molecule detection in capillary electrophoresis: Molecular shot noise as a fundamental limit to chemical analysis. Anal. Chem. 68, 690-696.

\section{TX800419P}

